\title{
LOW RESIDUAL NOISE SPEECH ENHANCEMENT UTILIZING TIME-FREQUENCY FILTERING
}

\author{
Gary Whipple \\ Department of Defense, 9800 Savage Road, Fort Meade, Maryland 20755 USA
}

\begin{abstract}
Spectral subtraction is a well known technique for enhancing speech corrupted by additive wideband noise. In this technique, the "clean" signal is approximated by subtracting a noise estimate from the spectrum of the corrupted signal. A negative side effect is the residual "musical" noise that is produced when isolated spectral peaks exceed the noise estimate. In this paper, a low residual noise enhancement method is presented. This method is based on spectral subtraction but incorporates an algorithm developed to suppress "musical" noise without affecting speech. The algorithm is referred to as time-frequency filtering because spectral peaks due to noise are eliminated on the basis of duration, bandwidth, and proximity to other peaks. Results showing the effects of combining spectral subtraction and time-frequency filtering are given.
\end{abstract}

\section{INTRODUCTION}

The corruption of speech by additive wideband noise is a common problem in communication systems and applications where voice input is required. In the past, many techniques for the enhancement of noisy speech signals have been developed. A well known class of techniques is based on subtracting a noise estimate from the original signal [1] - [5]

Subtractive techniques generally succeed in decreasing the noise level from that of the original signal but may fail to improve the overall quality. The lack of quality improvement is caused by the "musical" characteristics of the noise remaining in the processed signal. Listeners often find the "musical" noise in the processed signal more objectionable than the noise in the original signal.

In subtractive techniques, the subtraction of the noise estimate is typically performed in the spectral domain and in some cases the cepstral domain. Short duration tone bursts are produced when isolated peaks exceed the noise estimate. Noise with "musical" qualities is the result of the combination of many tones occurring at random frequencies in each analysis frame.

There have been previous efforts to improve the quality of speech processed by a subtractive algorithm. These efforts have focused on modifying the characteris- tics of the residual noise to make it more tolerable to the listener. Signal attenuation during non-speech activity, frame to frame spectral averaging, and another technique for reducing spectral fluctuations from frame to frame were proposed by Boll [1] as methods of reducing the negative effects of the residual noise components. A spectral floor was proposed by Berouti et. al. [2] as a technique to whiten the spectrum of the processed signal thereby masking the residual noise.

The emphasis of this work is to remove the residual noise components from the signal, without affecting the speech components. In this paper, an algorithm for filtering out the "musical" noise generated by subtractive enhancement techniques is described. The algorithm is known as time-frequency filtering and is described in detail in Section 2. In Section 3, spectral subtraction is combined with time-frequency filtering to create a low residual noise speech enhancement method. Results from a test of the enhancement method are presented in Section 4. Conclusions and directions for future work on this method are discussed in Section 5.

\section{TIME-FREQUENCY FILTERING}

In order to improve the quality of speech enhanced by spectral subtraction, an algorithm was formulated to remove the "musical" noise from the signal. This algorithm operates by analyzing several consecutive magnitude spectra and exploiting the differences in the spectral characteristics of speech and noise. Peaks that appear in several successive spectra, occupy a wide bandwidth, or are close to other spectral peaks are likely due to speech and are unmodified by the algorithm. Isolated spectral peaks are typically due to noise and are removed by the algorithm. The algorithm is referred to as time-frequency filtering because spectral peaks due to noise are eliminated on the basis of duration, bandwidth, and proximity to other peaks.

Time-frequency filtering is performed using several frames preceding and several frames following the frame(s) of interest. Let $X_{i}\left(e^{j \omega}\right)$ represent the magnitude spectrum of $x_{i}(t)$, where $x_{i}(t)$ is the $i$ th windowed frame of the signal. The area of analysis is defined by the two regions shown in Figure 1, region $A$ and region $B$. A decision on whether a noise peak or a speech peak is con- 


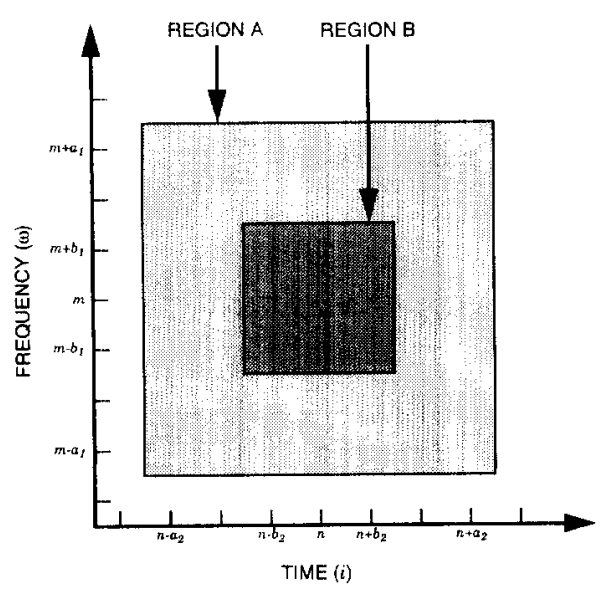

Figure 1: Analysis regions

tained in region $B$ is made using the ratio of the energies in regions $A$ and $B$. The energies contained in regions $A$ and $\mathrm{B}, P_{A}$ and $P_{B}$, are computed using the following,

$$
\begin{aligned}
& P_{B}(m, n)=\sum_{i=n-b_{2}, \omega=m-b_{1}}^{n+b_{2}} \sum_{i}^{m+b_{1}}\left(e^{j \omega}\right) \\
& P_{A}(m, n)=\sum_{i=n-a_{2} \omega}^{n+a_{2}} \sum_{m \sim a_{1}}^{m+a_{1}} X_{i}\left(e^{j \omega}\right)-P_{B}(m, n)
\end{aligned}
$$

where the values of $m$ and $n$ determine the center of the regions $A$ and $B$. The height and width of regions $A$ and $\mathrm{B}$ are determined by the values of $a_{1}, a_{2}, b_{1}$, and $b_{2}$. The decision as to whether or not region $B$ contains an isolated spectral peak is made according to the rules,

$$
\begin{aligned}
& \text { if } P_{B}(m, n) \geq \alpha \cdot P_{A}(m, n) \text {, then } \\
& \quad \text { region } B \text { contains an isolated peak } \\
& \text { if } P_{B}(m, n)<\alpha \cdot P_{A}(m, n) \text {, then } \\
& \text { region } B \text { does not contain an isolated peak }
\end{aligned}
$$

where $\alpha$ specifies the ratio of energies in regions $A$ and $B$. If region $B$ is found to contain an isolated spectral peak then the portions of the magnitude spectra contained in region $\mathbf{B}$ are modified according to,

$$
X_{i}\left(e^{j(1)}\right)=0
$$

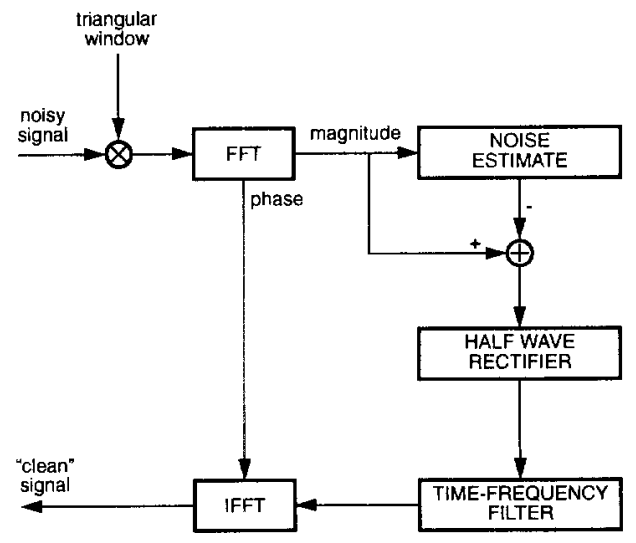

Figure 2: Simplified block diagram of the enhancement method

where $i=n-b_{2}, \ldots, n+b_{2}$ and $\omega=m-b_{1}, \ldots, m+b_{1}$. If region $B$ is found to not contain an isolated spectral peak, the portions of the magnitude spectra contained in region $B$ are left unmodified. For each frame in the signal, the center frequency of the analysis regions is varied to eliminate noise over the desired frequency range.

\section{ENHANCEMENT METHOD OVERVIEW}

In order to create a low residual noise speech enhancement method, the time-frequency filtering algorithm developed in the previous section is combined with a spectral subtraction algorithm. A simplified block diagram of the resulting enhancement method is shown in Figure 2.

The incoming signal is divided into frames which overlap each other by $50 \%$. Each frame is weighted by a triangular window and transformed using an FFT to produce the complex spectrum. The complex spectrum is separated into the magnitude spectrum and the phase information. Phase information is saved for use in reconstructing the time domain signal. The "clean" signal is estimated by subtracting the noise estimate from the magnitude spectrum. The noise estimate is updated using the magnitude spectrum during periods in the signal when no speech is present. The estimate of the "clean" signal is half wave rectified to remove any negative values that may result from the subtraction of the noise estimate. The magnitude spectrum of the current frame is combined with the magnitude spectra of several previous frames to produce a spectrogram representation of the signal. The time-frequency filtering algorithm developed in Section 2 is applied to the spectrogram to remove residual noise from the subtraction processing. 


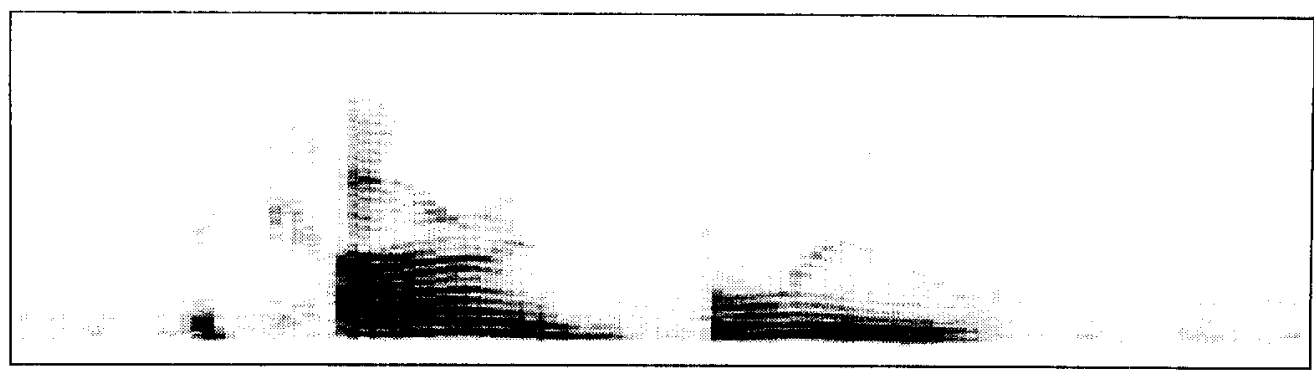

Figure 4a: Original speech signal

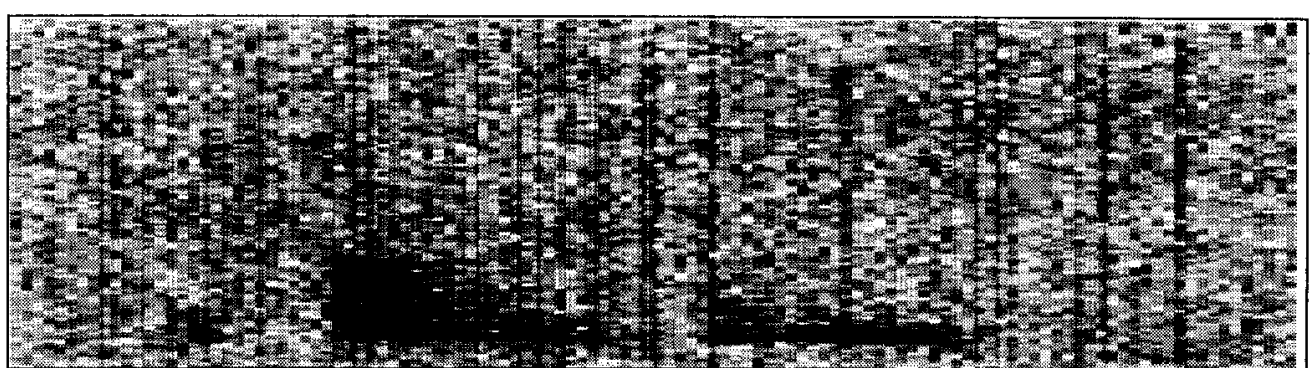

Figure 4b: Original speech signal with noise added

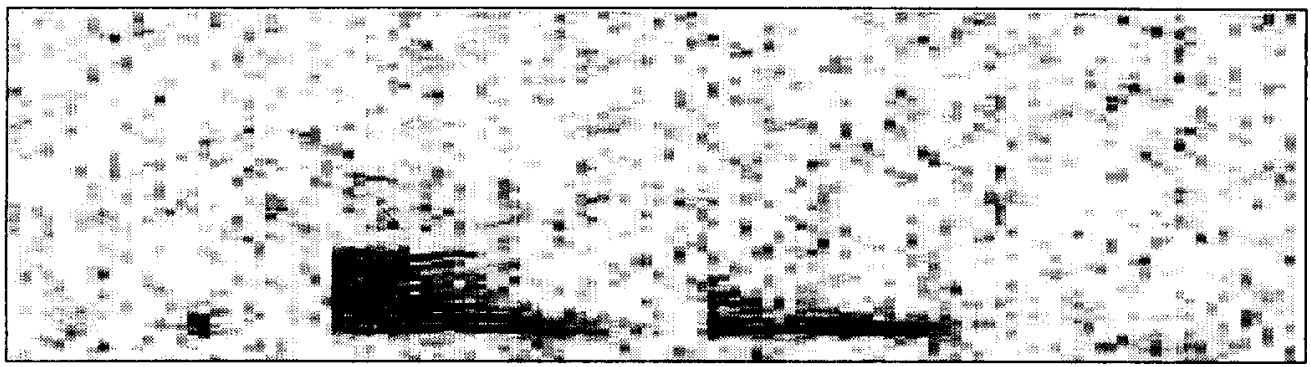

Figure 4c: Noisy speech signal processed by spectral subtraction alone

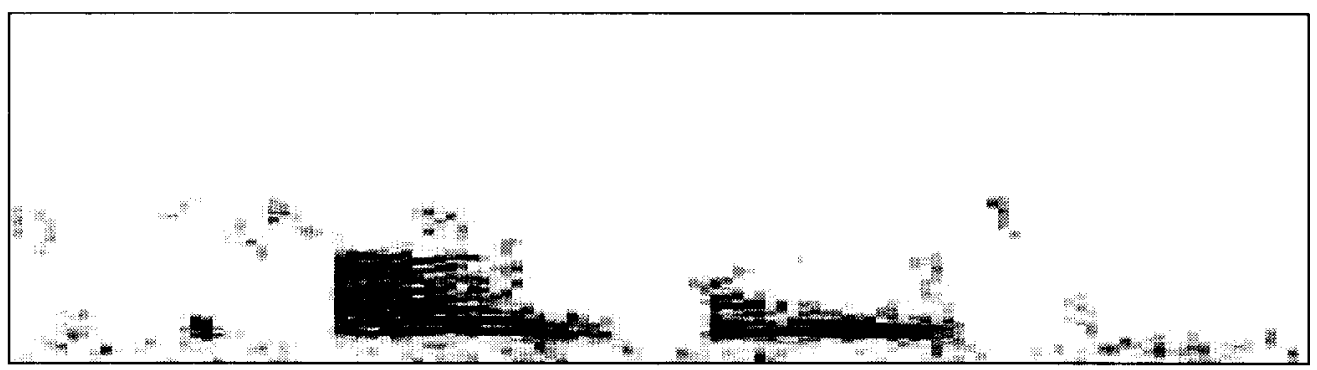

Figure 4d: Noisy speech signal processed by spectral subtraction and time-frequency filtering 
Following the time-frequency filtering step, the oldest magnitude spectrum is removed from the spectrogram. This magnitude spectrum is combined with the stored phase information from the proper frame to form the complex spectrum. The time domain signal is then reconstructed using an inverse FFT,

\section{RESULTS}

For test purposes, a corrupted speech signal was created by adding white Gaussian noise to speech recorded digitally at a sampling rate of $8 \mathrm{kHz}$. Spectrograms of the original and corrupted signals are shown in Figures 4a and $4 \mathrm{~b}$, respectively. The test signal was processed using spectral subtraction alone and using spectral subtraction followed by time-frequency filtering. A frame length of 128 samples $(16 \mathrm{~ms})$ was used for all of the processing. For the time-frequency filtering, a value of 10 was used for $\alpha$ (see Eq. 3 ) and the dimensions of the analysis regions were specified by

$$
\begin{aligned}
& a_{1}=3, a_{2}=3 \\
& b_{1}=2, b_{2}=2
\end{aligned} .
$$

Figure $4 \mathrm{c}$ shows the spectrogram of the speech processed using spectral subtraction alone. Although some noise has been removed, a substantial amount remains. The spectrogram in Figure 4d show results of processing the test signal with spectral subtraction followed by time-frequency filtering. Most of the noise that remained in the signal processed by spectral subtraction has been removed without significantly affecting the speech. An informal listening test confirmed that the time-frequency filtering had significantly improved the quality of the enhanced signal.

\section{CONCLUSIONS}

A low residual noise speech enhancement method has been presented. The method utilizes a time-frequency filtering algorithm to remove the residual noise produced by spectral subtraction. An informal listening test indicates the method can substantially reduce the level of "musical" noise in speech enhanced by subtractive techniques.

Future work will concentrate on improvements in the time-frequency filtering algorithm. Possibilities include varying the shape of the analysis regions and experimenting with a variety of spectral estimation techniques.

\section{REFERENCES}

[1] S. Boll, "Suppression of Acoustic Noise in Speech Using Spectral Subtraction," IEEE Trans. on ASSP, Vol. ASSP-27, pp. 113-120, April 1979.

[2] M. Berouti, R. Schwartz, and J. Makhoul, "Enhancement of Speech Corrupted by Acoustic Noise," Proceedings of the IEEE 1979 International Conference on Acoustics, Speech, and Signal Processing, pp. 208-211, April 1979.

[3] Kushner, W.M., Goncharoff, V., Wu, C., Nguyen, V., Damoulakis, J.N., "The Effects of Subtractive-Type Speech Enhancement/Noise Reduction Algorithms on Parameter Estimation for Improved Recognition and Coding in High Noise Environments," Proceedings of the IEEE 1989 International Conference on Acoustics, Speech and Signal Processing, pp. 211214, May 1989.

[4] M. R. Weiss, E. Aschkenasy, and T. W. Parsons, "Study and Development of the INTEL Technique for Improving Speech Intelligibility," Nicolet Scientific Corp., Final. Tech. Rep. RADC-TR-75-108.

[5] J. S. Lim and A. V. Oppenheim, "Enhancement and Bandwidth Compression of Noisy Speech," Proceedings of the IEEE, Vol. 67, No. 12, pp. 1586-1604, December 1979 . 